
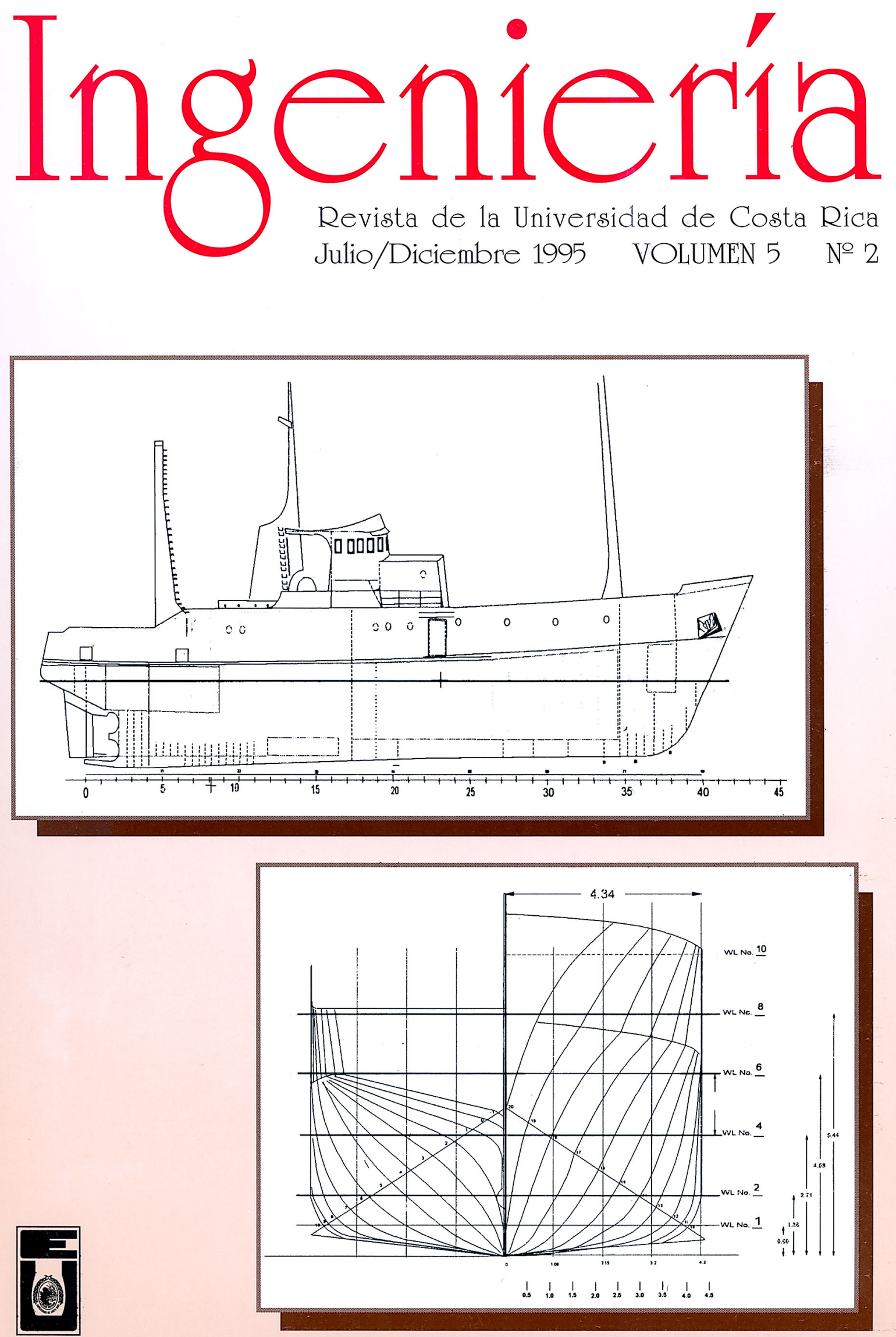


\title{
METODOLOGÍA PARA EL ESTUDIO DE CONDICIONES EXTERIORES DE DISEÑO PARA ESTIMACIONES DE LA CARGA DE ENFRIAMIENTO EN LOCALIDADES COSTARRICENSES
}

\author{
Juan José Gutiérrez S. *
}

\begin{abstract}
RESUMEN
Se explica la metodología utilizada en el estudio de condiciones meteorológicas que afectan el diseño de sistemas de aire acondicionado en Costa Rica. Para efectos ilustrativos se indican algunos resultados típicos obtenidos; en artículos posteriores se describirán en detalle los resultados para diferentes lugares del país.
\end{abstract}

\section{SUMMARY}

The procedure used in the study of meteorological conditions which affect the design of air conditioning systems in Costa Rica is described. For illustrative purposes some typical results are shown. Detailed results for different parts of the country will be described in future articles.

\section{1- INTRODUCCION}

El empleo de acondicionamiento del aire en edificios en Costa Rica ha crecido mucho en tamaño y complejidad durante los últimos años; es de esperar que, con el crecimiento de las ciudades y el mayor desarrollo de actividades turísticas e industriales, se instalen en nuestro medio sistemas cada vez más grandes y complejos. El diseño y operación correctos y económicos de sistemas de climatización interna requiere el conocimiento de factores ambientales exteriores al edificio que afectan el dimensionamiento del sistema y la selección de sus componentes. Hasta la fecha, en Costa Rica, tal información no estaba disponible pues no habían sido realizados estudios rigurosos al respecto. Esta publicación pretende divulgar información obtenida sobre el tema mediante procedimientos y objetivos centrados más en aplicaciones de ingeniería que en aspectos puramente estadísticos.

Se ha observado que los estudios realizados tienen también aplicación en el diseño de otro tipo

Ing. Mec., M.Sc., Profesor, Escuela de Ingeniería Mecánica, Universidad de Costa Rica.

Ing. Consultor. de sistemas térmicos (por ejemplo calentamiento o enfriamiento de fluidos almacenados en tanques, sistemas de refrigeración) y aplicaciones agrícolas y apícolas.

\section{2- LA CARGA DE ENFRIAMIENTO}

\section{2-1 Cargas pico y parcial}

Los métodos de cálculo de la carga de enfriamiento han ido evolucionando y tendiendo a una mayor perfección, desde métodos simples que requieren el uso de tablas, gráficos y calculadora hasta métodos más sofisticados que demandan programas de computadora. Los métodos sencillos requieren definir un valor de la variable tiempo para el cual se harán los cálculos (hora, fecha, mes). Los programas de computadora permiten usualmente hacer el cálculo para cualquier hora del año. La precisión y velocidad de cálculo, el costo y tamaño del sistema a diseñar, y cuán rigurosos sean sus requerimientos, son variables que determinan el tipo de método que se debe utilizar.

Al calcular la carga de enfriamiento, el diseñador puede hacerlo con diferentes propósitos, los 
cuales determinan el "volumen de control" a utilizar y el valor de la variable tiempo (fecha y hora de diseño) a utilizar. Cada volumen de control posee características constructivas particulares que determinan la forma en que la combinación de componentes de carga lo afectan, por esta razón no es posible dar reglas absolutas para definir en qué momento hay que hacer el calculo de carga; cada situación debe ser analizada individualmente. En algunos casos se trata de determinar un valor máximo de carga (carga pico) con el objeto de seleccionar un equipo que tenga la capacidad de absorber siempre esa carga. La carga pico puede ser estimada para una zona, para toda una edificación o conjunto de edificaciones (carga bloque) compuesto de varias zonas. Una sobreestimación o una subestimación de la carga de enfriamiento puede tener resultados perjudiciales en cuanto a costos, eficiencia y operación del sistema de aire acondicionado.

A veces se estiman cargas parciales para hacer análisis de aumento de humedad en el espacio acondicionado o con el objeto de utilizarlas en procedimientos de simulación que estiman consumos de energía o analizan valores de calor de rechazo que puede ser recuperado.

\section{2-2 Condiciones internas de diseño}

La evaluación de la carga de enfriamiento depende de las condiciones de diseño que se deben mantener en el recinto acondicionado. La selección de estos valores se hace con la finalidad de satisfacer los requerimientos de los ocupantes, equipo o proceso para los cuales se efectúa el acondicionamiento del aire. Dependiendo de cuán crítica sea la aplicación se permitirá una tolerancia en la variación de las condiciones internas, en mayor o menor grado.

\section{2-3 Condiciones externas de diseño}

Las condiciones externas de diseño están relacionadas con componentes de la carga perimetral o carga por aire exterior y normalmente requieren definición de las siguientes variables: finida mediante dos variables coincidentes, que den indicación del contenido de energía y masa (humedad). Se utilizan usualmente temperatura de bulbo seco y temperatura de bulbo húmedo.

b- El ámbito diario de variación de la temperatura de bulbo seco por cuanto los valores de la carga en un instante son afectados por la sucesión de valores en intervalos de tiempo anteriores.

c- Radiación solar incidente, afectada por claridad de la atmósfera, incluyendo posible nubosidad.

d- Velocidad del viento en cuanto afecta a los coeficientes de convección sobre superficies exteriores y la posible infiltración de aire al recinto acondicionado.

e- La latitud es una variable de importancia pues afecta significativamente las fluctuaciones en radiación solar, temperaturas de bulbo seco y parámetros relacionados con la humedad. En países de latitud alta existen fluctuaciones significativas a lo largo del año en la radiación y las condiciones psicrometricas del aire exterior.

En la evaluación de la carga de enfriamiento se hace también la suposición de que el clima se repite en forma cíclica en un período anual y en ciclos diarios durante períodos más cortos.

Para cálculos de tipo manual y evaluación de cargas pico o parciales, las condiciones exteriores de diseño son, usualmente, definidas con el objetivo final de seleccionar aquéllas que correspondan a un momento determinado en el cual la combinación de componentes de la carga produzca la situación de cálculo más crítica. La selección de ese momento se hace dentro de un grupo de meses determinado, en los cuales la probabilidad de que se den las condicionesde cálculo más críticas es muy alta. En países de latitudes altas el grupo de meses es reducido; por ejemplo en la mayoría de los Estados Unidos de Norteamérica las condiciones de diseño para carga de enfriamiento se definen estudiando el clima de los cuatro meses más calientes del año; en Canadá, lo hacen estudiando el mes más caliente. Los países de latitudes bajas o de fluctuaciones de clima moderadas por la presencia de otros factores (como la cercanía del mar) presentan la posibilidad 
de que en muchos meses del año se den condiciones que puedan producir cargas pico; en estos casos se prefiere definir condiciones para diseño con base en un examen del clima a lo largo del todo el año; este es el caso de Costa Rica.

El empleo de ordenadores para el cálculo de cargas de enfriamiento requiere conocer la variación horaria de las condiciones exteriores en un día típico de cálculo; el uso de esos equipos para análisis de consumo de energía requiere conocer el comportamiento horario promedio en cada uno de los meses del año.

La cercanía al mar, la presencia de montañas que generen microclimas, la existencia de llanuras que permiten que efectos meteorológicos afecten grandes extensiones, hacen que la única forma de definir condiciones típicas para el diseño en aire acondicionado sea mediante el estudio estadístico de registros climatológicos. No es posible, por el momento, predecir teóricamente dichas condiciones, pero sí es factible obtener correlaciones con base en los registros estudiados.

\section{3- ESTUDIOS PARA COSTA RICA}

La revisión de literatura sobre el tema mostró únicamente algunos valores sugeridos para el diseño de aire acondicionado en San José (Marley, 1973). En años recientes se han estudiado para Costa Rica los registros meteorológicos de algunos lugares de interés, ya sea por su población y por su actividad industrial o turística, con la intención de ir definiendo condiciones climatológicas aplicables al diseño en aire acondicionado. Por limitaciones de información disponible, de su confiabilidad, o procedimientos de análisis, se ha dado prioridad al estudio de las temperaturas y la humedad del ambiente; posteriormente, se podrían agregar estudios de radiación solar (junto con nubosidad presente) o velocidad de viento para diseño de aire acondicionado. Por el momento hay resultados disponibles para lugares indicados en la Tabla $\mathrm{N}^{\circ} 1$ y se encuentra en proceso el estudio de la estación de Sabanilla, en San José.

Por las características montañosas de nuestro país se generan microclimas locales que requieren estudio individual. En el Instituto Meteoroógico Nacional (IMN) existen datos que, debidamente procesados, darían información de gran utilidad para aplicaciones de acondicionamiento de aire en zonas de importancia.

\section{4- PROCEDIMIENTO}

\section{4-1 Generalidades}

El IMN registra en algunas estaciones meteorológicas valores horarios de temperatura de bulbo seco y de humedad relativa mediante termohidrógrafos (y recientemente mediante registradores digitales); esta información es anotada en formularios especiales y recientemente ha comenzado a ser grabada en disquetes para computadora. Se desarrollaron programas especiales que procesan dicha información y obtienen parámetros útiles para cálculos de acondicionamiento de aire; dichos programas consideran el efecto de la elevación sobre el nivel del mar.

\section{4-2 Tamaño de la muestra}

Si se parte de la suposición de que la muestra estudiada debería mostrar una distribución normal y de que los ciclos anuales se repiten, una muestra suficiente para evaluar condiciones de diseño debería ser de unos tres años, desde el punto de vista estadístico (Ramirez 1990). El análisis de las muestras estudiadas indicó que su distribución no era perfectamente normal en algunos casos (Bolaños 1986); si a esto se le agrega el hecho de que existen factores que hacen que los ciclos anuales se vean alterados de vez en cuando (fenómeno del Niño, etc.) se consideró prudente extender el tamaño de las muestras. Comparando resultados con diferentes números de años de tamaño de muestra, se considera que cinco años es un mínimo prudente para usos normales en aire acondicionado como obtención de porcentajes de diseño o curvas horarias para cálculos de cargas; dependiendo de la aplicación de los resultados, el tamaño de la muestra requerida puede variar.

\section{4-3 Revisión de datos y resultados para cada estación}

El IMN indicó que los instrumentos estaban correctamente calibrados durante los períodos 
estudiados, por lo que se puede suponer que operaban dentro de las tolerancias especificadas por la Organización Meteorológica Mundial: temperaturas de bulbo seco $0,1{ }^{\circ} \mathrm{C}$., humedad relativa $3 \%$. (Bolaños 1986). Los datos de temperaturas y humedades suministrados a los programas fueron revisados comparando los registros originales del IMN con hojas de impresión de la muestra recibida por la computadora. Los programas hechos eran capaces de reconocer y eliminar de la muestra datos considerados incongruentes. Los resultados obtenidos fueron revisados manualmente en forma aleatoria.

\section{4-4 Parámetros a obtener}

Con base en parámetros sugeridos por la literatura revisada y el examen de las necesidades de los procedimientos de cálculo de carga de enfriamiento, se consideró importante obtener los siguientes parámetros o grupos de ellos:

\section{a- Tabla de frecuencias}

Es un ordenamiento en el cual se muestra, para cada mes del año, el número de veces que se ha registrado un determinado valor de una variable en todo el intervalo de tiempo estudiado. En este trabajo se ha realizado para temperaturas de bulbo seco en todas las estaciones y programas en desarrollo tienden a lograrlo para temperaturas de bulbo húmedo.

La disponibilidad de tablas de frecuencia de bulbo seco es útil para observar cuáles son los meses más calientes o en cuáles de ellos se presentan las temperaturas seleccionadas con base en porcentajes de diseño.

Las gráficas de los termohidrógrafos del IMN utilizados fueron leídas visualmente al décimo de grado centígrado para las temperaturas de bulbo seco por personal de dicha institución y los valores anotados en formularios especiales. Para la elaboración de las tablas de frecuencia se consideró que era difícil esperar tal precisión visual debido al tamaño de las bandas de registro que usa el instrumento y que era más conveniete redondear los valores a un incremento mayor; se escogió agruparlos en intervalos de clase de medio grado, con centros de incremento en valores enteros o de medios grados. (Ver tabla $\mathrm{N}^{\mathrm{o}} 2$ ).

\section{b- Porcentaje de diseño para temperaturas de bulbo seco (\%tbs)}

Es el porcentaje, respecto al total de horas de un año, con el cual se espera que las temperaturas de bulbo seco igualen o excedan el valor seleccionado de la tabla de frecuencias, ya sea para aplicaciones de enfriamiento del aire o para calentamiento del mismo. El "porcentaje nominal" se basa en la escogencia de un número sencillo (ej: $0,1 \%$ ) y en 8760 horas por año. El "porcentaje real" considera el hecho de que el número de horas estudiadas en el período puede ser menor que el total ideal, debido a que puede haber datos faltantes o eliminados por el programa al considerarlos incongruentes.

El uso de un valor determinado de porcentaje depende del costo del equipo y la tolerancia permitida en el mantenimiento de las condiciones de diseño. Se definen valores que produzcan cambios significativos en tamaños comerciales de equipos. Se sugieren las siguientes guías en su uso, para aplicaciones de enfriamiento del recinto:

1- Un número bajo de horas al año en que la temperatura es igualada o excedida (por ejemplo 9 horas correespondientes a un porcentaje de $0,1 \%$ ) para trabajo conservador (instalaciones muy sensitivas, etc).

2- $\quad$ Trabajo promedio: $0,5 \%, 44$ horas al año.

3- Trabajo de promoción, en donde el costo es más importante que el mantenimiento exacto de las condiciones internas: $2 \%, 175$ horas al año.

\section{c- Ambito diario promedio para un mes}

En este artículo está definido como la diferencia entre el promedio de las temperaturas de bulbo seco horarias máximas diarias (ThMd) y el promedio de las temperaturas horarias mínimas diarias (Thmd) para el mes correspondiente. 
Tabla No. 2

Frecuencias de Temperatura de

Bulbo Seco $\left({ }^{\circ} \mathrm{C}\right)$ para Pavas.

(seis años)

TEMP Ene Feb Mar Abr May Jun Jul Ago Set Oct Nov Dic TOT TOT.A \%Rea

\begin{tabular}{|c|c|c|c|c|c|c|c|c|c|c|c|c|c|c|c|}
\hline 32.0 & & & & & & & & & & & & & 0 & 0 & 0.00 \\
\hline 31.5 & & & 3 & 2 & & & & & & & & & 5 & 5 & 0.01 \\
\hline 31.0 & & & 4 & 2 & & & & & & & & & 6 & 11 & 0.02 \\
\hline 30.5 & & & 7 & 6 & & & & & & & & & 13 & 24 & 0.05 \\
\hline 30.0 & & 2 & 23 & 38 & 9 & & & & & & & & 72 & 96 & 0.18 \\
\hline 29.5 & 1 & 5 & 27 & 41 & 11 & & & & 1 & & 2 & & 88 & 184 & 0.35 \\
\hline 29.0 & 2 & 20 & 65 & 80 & 36 & 4 & 1 & 6 & 4 & 1 & 3 & & 222 & 406 & 0.77 \\
\hline 28.5 & 2 & 22 & 70 & 98 & 60 & 14 & 6 & 7 & 15 & 1 & 2 & 3 & 300 & 706 & 1.34 \\
\hline 28.0 & 13 & 45 & 111 & 137 & 102 & 50 & 25 & 26 & 27 & 23 & 16 & 10 & 585 & 1291 & 2.46 \\
\hline 27.5 & 15 & 36 & 82 & 109 & 85 & 51 & 34 & 36 & 46 & 30 & 8 & 10 & 542 & 1833 & 3.49 \\
\hline 27.0 & 47 & 97 & 137 & 150 & 144 & 100 & 69 & 74 & 83 & 68 & 60 & 27 & 1056 & 2889 & 5.50 \\
\hline 26.5 & 67 & 58 & 99 & 114 & 115 & 115 & 87 & 86 & 72 & 78 & 72 & 44 & 1007 & 3896 & 7.41 \\
\hline 26.0 & 98 & 148 & 128 & 142 & 154 & 164 & 153 & 135 & 126 & 170 & 138 & 123 & 1679 & 5575 & 10.61 \\
\hline 25.5 & 91 & 109 & 112 & 109 & 102 & 105 & 117 & 116 & 117 & 115 & 108 & 100 & 1301 & 6876 & 13.08 \\
\hline 25.0 & 175 & 194 & 179 & 162 & 174 & 165 & 183 & 189 & 178 & 174 & 226 & 182 & 2181 & 9057 & 17.23 \\
\hline 24.5 & 141 & 100 & 119 & 99 & 85 & 114 & 133 & 110 & 106 & 91 & 123 & 143 & 1364 & 10421 & 19.83 \\
\hline 24.0 & 187 & 168 & 148 & 149 & 161 & 139 & 199 & 205 & 134 & 151 & 178 & 213 & 2032 & 12453 & 23.69 \\
\hline 23.5 & 158 & 117 & 116 & 118 & 128 & 140 & 129 & 99 & 102 & 112 & 115 & 166 & 1500 & 13953 & 26.55 \\
\hline 23.0 & 204 & 158 & 160 & 184 & 235 & 168 & 231 & 179 & 176 & 151 & 207 & 282 & 2335 & 16288 & 30.99 \\
\hline 22.5 & 173 & 130 & 146 & 138 & 165 & 178 & 163 & 154 & 124 & 114 & 139 & 214 & 1838 & 18126 & 34.49 \\
\hline 22.0 & 176 & 176 & 209 & 288 & 327 & 290 & 296 & 283 & 211 & 187 & 286 & 354 & 3083 & 21209 & 40.35 \\
\hline 21.5 & 165 & 164 & 177 & 268 & 257 & 259 & 266 & 229 & 159 & 163 & 197 & 305 & 2609 & 23818 & 45.31 \\
\hline 21.0 & 266 & 281 & 280 & 442 & 433 & 462 & 438 & 465 & 266 & 334 & 395 & 350 & 4412 & 28230 & 53.71 \\
\hline 20.5 & 237 & 198 & 213 & 308 & 301 & 287 & 371 & 364 & 270 & 299 & 262 & 267 & 3377 & 31607 & 60.13 \\
\hline 20.0 & 417 & 397 & 398 & 451 & 437 & 532 & 575 & 578 & 517 & 675 & 522 & 482 & 5981 & 37588 & 71.51 \\
\hline 19.5 & 279 & 267 & 290 & 216 & 212 & 249 & 280 & 287 & 374 & 380 & 329 & 297 & 3460 & 41048 & 78.10 \\
\hline 19.0 & 532 & 402 & 408 & 235 & 271 & 277 & 326 & 337 & 490 & 454 & 413 & 345 & 4490 & 45538 & 86.64 \\
\hline 18.5 & 351 & 234 & 236 & 93 & 157 & 174 & 148 & 204 & 292 & 229 & 193 & 238 & 2549 & 48087 & 91.49 \\
\hline 18.0 & 338 & 259 & 215 & 68 & 133 & 155 & 121 & 146 & 248 & 227 & 173 & 163 & 2246 & 50333 & 95.76 \\
\hline 17.5 & 142 & 92 & 115 & 28 & 59 & 63 & 43 & 65 & 79 & 105 & 47 & 63 & 901 & 51234 & 97.48 \\
\hline 17.0 & 107 & 113 & 97 & 28 & 62 & 42 & 42 & 51 & 48 & 73 & 54 & 45 & 762 & 51996 & 98.93 \\
\hline 16.5 & 27 & 29 & 30 & 13 & 23 & 13 & 8 & 12 & 20 & 32 & 24 & 20 & 251 & 52247 & 99.40 \\
\hline 16.0 & 29 & 27 & 33 & 1 & 8 & 4 & 8 & 17 & 14 & 14 & 11 & 11 & 177 & 52424 & 99.74 \\
\hline 15.5 & 9 & 16 & 7 & 1 & 5 & 2 & 1 & & 6 & 6 & 5 & 1 & 59 & 52483 & 99.85 \\
\hline 15.0 & 8 & 10 & 16 & 1 & 3 & & 3 & 2 & 6 & 1 & & 5 & 55 & 52538 & 99.96 \\
\hline 14.5 & 2 & 2 & & & & & & & 1 & & 1 & 1 & 7 & 52545 & 99.97 \\
\hline 14.0 & & 3 & 3 & 1 & & & & & 2 & & & & 9 & 52554 & 99.99 \\
\hline 13.5 & 4 & 1 & & & & & & & & & & & 5 & 52559 & 100.0 \\
\hline 13.0 & 1 & & 1 & & & & & & & & & & 2 & 52561 & 100.0 \\
\hline
\end{tabular}




$$
\text { Adpm }=\frac{\text { ThMd }- \text { Thmd }}{\text { Ndm }}
$$

$\mathrm{Ndm}=$ Número de días en el mes estudiado con registros aceptados de temperaturas de bulbo seco.

El ámbito diario tiene importancia pues afecta las correcciones a hacer a ciertas variables de cálculo relacionadas con diferencias de temperaturas a través de losas, paredes, etc. Alguna literatura (Carrier, Systems Design Manual) lo evalúa únicamente para el mes más caliente del año.

\section{d- $\quad$ Temperatura de bulbo seco horaria}

Es el valor de temperatura de bulbo seco, registrado en un instante del día que corresponde a una hora que es un número entero (Ej: 6.00, 15.00.). Al no considerarse registros en instantes intermedios (Ej: 6.15 ó 15.08), la temperatura máxima o mínima del día puede diferir un poco de la temperatura máxima horaria o temperatura mínima horaria para ese mismo día.

Posteriormente se harán comentarios sobre la forma que toman las curvas de temperaturas horarias en Costa Rica y la forma que supone ASHRAE (American Society of Heating, Refrigerating and Air Conditioning Engineers, Inc) en su método de cálculo.

El estado termodinámico del aire exterior en un "momento" dado (hora y fecha en este trabajo) debe ser definido mediante el valor coincidente en ese momento de dos variables independientes entre sí.

\section{e- Humedad relativa coincidente}

Es el valor promedio de las humedades relativas coincidentes con un valor de temperatura de bulbo seco.

\section{f- Temperatura de bulbo húmedo coincidente}

Es el valor promedio de las temperaturas de bulbo húmedo coincidentes con un valor de temperatura de bulbo seco. La literatura cita métodos para estimar la curva horaria de esta variable si se conocen el "ambito diario", el valor de bulbo seco de diseño y se supone que el punto de rocío del aire exterior permanece constante (Carrier, Systems Design Manual; Carrier, E-20). Con los resultados de humedad relativa coincidente logrados en este trabajo, puede ser obtenida fácilmente.

Para análisis de enfriamiento evaporativo es útil obtener porcentajes de diseño para temperaturas de bulbo húmedo y el valor coincidente de otra variable independiente.

\section{g- Epoca caliente}

Es el conjunto de los cuatro meses con los promedios mensuales más altos de temperatura de bulbo seco.

Puede ayudar a definir una hora de diseño para cálculos de tipo manual.

\section{h- Epoca fría}

Es el conjunto de los tres meses con los promedios mensuales más bajos de temperatura de bulbo seco.

Puede ayudar a definir una hora de diseño para cálculos de tipo manual.

\section{i- Día típico caliente}

Está descrito mediante las curvas horarias de dos variables independientes que describan el estado psicrométrico del aire en el ambiente exterior, las cuales se obtienen como sigue:

- $\quad$ Los días más calientes quedan definidos como aquéllos que tienen el mayor promedio diario de temperaturas horarias de bulbo seco.

- Para un mes se seleccionan los "n" días más calientes y se promedian los valores horarios de temperatura de bulbo seco en esos n días.

- Para la segunda variable, se determinan los valores horarios coincidentes con la primera variable y se promedian por hora.

Las curvas horarias obtenidas son útiles para estimar cargas pico de enfriamiento con programas de computador, para cualquier mes del año. (Ver Tabla $\mathrm{N}^{\circ} 3$ y Figura $\mathrm{N}^{\circ} 1$ ). 
Tabla $N^{0} 3$

Día Típico Caliente

Estación 84072, Pavas, mes de abril (1)

\begin{tabular}{|c|c|c|c|c|}
\hline Hora & Bulbo seco & $\begin{array}{l}\text { Temperaturas }\left({ }^{\circ} \mathrm{C}\right) \\
\text { Bulbo húmedo }\end{array}$ & Punto de rocío & $\begin{array}{c}\text { Humedad } \\
\text { relativa } \\
\%\end{array}$ \\
\hline 1 & 20,4 & 17,9 & 16,8 & 79,8 \\
\hline 2 & 20,1 & 17,7 & 16,5 & 79,9 \\
\hline 3 & 19,9 & 17,5 & 16,3 & 79,7 \\
\hline 4 & 19,7 & 17,3 & 16,1 & 79,6 \\
\hline 5 & 19,5 & 17,0 & 15,8 & 79,1 \\
\hline 6 & 19,7 & 17,0 & 15,6 & 77,3 \\
\hline 7 & 21,9 & 17,8 & 15,9 & 68,6 \\
\hline 8 & 24,0 & 18,3 & 15,5 & 59,1 \\
\hline 9 & 26,0 & 18,9 & 15,6 & 52,7 \\
\hline 10 & 27,2 & 19,1 & 15,3 & 48,1 \\
\hline 11 & 28,4 & 19,5 & 15,5 & 45,5 \\
\hline 12 & 28,7 & 19,9 & 16,0 & 46,2 \\
\hline 13 & 29,0 & 20,4 & 16,6 & 47,2 \\
\hline 14 & 28,8 & 20,6 & 17,1 & 49,3 \\
\hline 15 & 27,8 & 20,5 & 17,4 & 53,1 \\
\hline 16 & 26,8 & 20,4 & 17,8 & 58,4 \\
\hline 17 & 25,1 & 20,3 & 18,3 & 65,9 \\
\hline 18 & 23,9 & 20,1 & 18,5 & 72,0 \\
\hline 19 & 22,7 & 19,8 & 18,6 & 77,9 \\
\hline 20 & 22,3 & 19,7 & 18,6 & 79,6 \\
\hline 21 & 21,9 & 19,5 & 18,5 & 80,9 \\
\hline 22 & 21,6 & 19,3 & 18,3 & 81,7 \\
\hline 23 & 21,2 & 18,9 & 17,9 & 81,7 \\
\hline 24 & 20,9 & 18,6 & 17,7 & 81,7 \\
\hline
\end{tabular}

(1) - Valores horarios de bulbo seco y humedad relativa promediados para los seis días más calientes del mes en toda la muestra. - Días más calientes definidos como aquellos con los mayores promedios diarios.

Por la información disponible para Costa Rica, se generaron curvas de temperatura de bulbo seco y humedad relativa. Los programas desarrollados pueden trabajar para un valor de "n" ajustable, con el objeto de escoger cuánto se diferencia el día "tipico caliente" del "día promedio" del mes. Este parámetro fue utilizado por primera vez en el estudio realizado para San José y Turrialba. (Bonilla,1989).

\section{j- $\quad$ Día típico frío}

Su definición es semejante a la anterior, excepto que resulta de seleccionar los "n" días más fríos del mes.

Las curvas obtenidas pueden ser útiles para cálculos (manuales o con computador) de calefacción, límite de humedad relativa interior con carga sensible parcial, o ciclos "economizadores".

\section{k- Curvas horarias promedio para cada mes}

Las curvas se obtienen para un mes determinado, promediando para cada hora del día, los valores de una variable. Se obtienen utilizando $\mathrm{n}=$ total de días del mes, en el programa para el día típico caliente. Para una segunda variable se determinan primero los valores horarios coincidentes y luego se promedian por hora.

$\mathrm{Si}$, por ejemplo, se contara con curvas horarias promedio para temperaturas de bulbo seco y humedad relativa exteriores, y programas que permitan calcular con ellas cualquier otra variable 
FIGURA No. 1

Día típico caliente estación 84072, Pavas, mes de abril

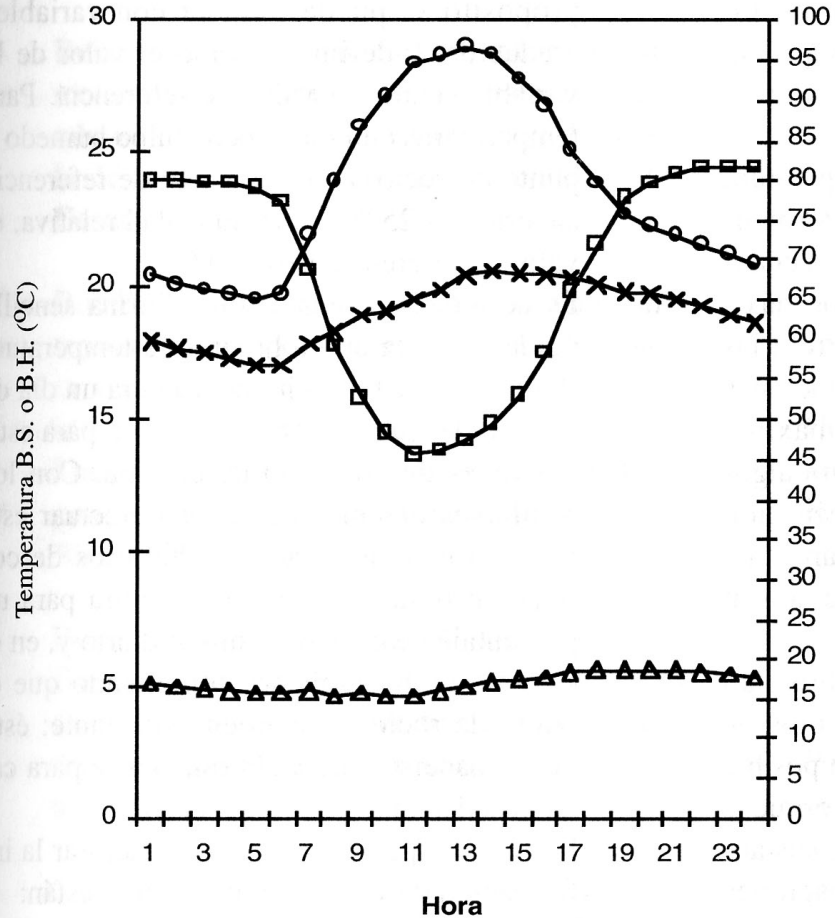

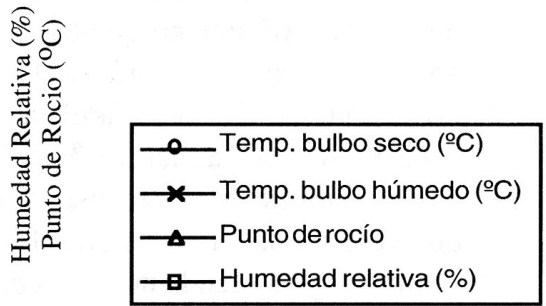

5- RESULTADOS

a- En este artículo se muestran algunos resultados obtenidos en cuanto a porcentajes de diseño, valores coincidentes de temperaturas de diseño, ámbito diario, distribución de frecuencias de temperaturas de bulbo seco, etc. únicamente para ilustrar los conceptos utilizados. En artículos posteriores se publicarán con mayor detalle resultados para cada lugar estudiado. Los resultados obtenidos permiten seleccionar con buen criterio el mes y la hora para estimaciones de cargas pico y usar curvas horarias para programas de carga pico o parcial.

b- Del estudio de las tablas de frecuencias de las diferentes estaciones (ejemplo, Tabla $\mathrm{N}^{\circ}$ 2) se observa que las temperaturas de diseño para enfriamiento de aire se presentan en general en muchos meses del año, por lo que 
es preferible estudiar la frecuencia de los valores sobre una base anual y no respecto a algunos de los meses más calientes como se hace en otros países.

c- En algunos casos se observaron distribuciones de temperaturas de bulbo seco que no corresponden exactamente a un a curva de distribución normal (Bolaños, 1986 )

d- $\quad$ La forma de la curva horaria de temperatura para un día de diseño difiere de la normalizada por ASHRAE, principalmente en cuanto a la hora en que se presenta el valor máximo. ASHRAE supone que esto ocurre a las 15 horas y en la mayoría de las estaciones estudiadas en Costa Rica el valor máximo ocurre alrededor de las 12 horas. Para algunas estaciones se ha obtenido una ecuación que describe la curva horaria. Las partes ascendentes y descendentes de la curva no son simétricas.

e- Cabe anotar que, aunque los estudios han sido realizados para su uso en cálculos de aire acondicionado, tienen aplicación posible en evaluaciones de otros sistemas térmicos como refrigeración, calentamiento de sustancias guardadas en tanques, y aplicaciones agrícolas y apícolas (para las cuales ya ha habido interés en los datos y resultados).

\section{6- RECOMENDACIONES}

Es útil comparar y completar resultados para las estaciones meteorológicas de la Gran Área Metropolitana (G.A.M.) de Costa Rica; actualmente hay resultados para San José centro (Aranjuez), Pavas y Aeropuerto Juan Santamaría. El estudio de datos para la estación ubicada en Sabanilla y alguna cerca de Cartago completaría este cuadro y permitiría estimar valores para otros sitios de la G.A.M. El estudio de procedimientos para interpolar resultados entre estaciones ayudaría en este aspecto.

b- Conviene estudiar también sitios en la costa oeste del Guanacaste, Zona Sur y Zona Norte en donde haya centros de población grandes o actividades turísticas o industriales de importancia. c- Podría ser útil obtener curvas "generalizadas" para hacer comparaciones entre diferentes lugares y meses, para describir matemáticamente ciertas curvas, etc. Con este propósito se puede trabajar con variables "reducidas", definidas como el valor de la variable entre un valor de referencia. Para temperaturas de bulbo seco, bulbo húmedo o punto de rocío, la temperatura de referencia sugerida es $25^{\circ} \mathrm{C}$; para humedad relativa, el valor de referencia sería $50 \%$.

d- Es de interés contar con una forma sencilla de describir la curva horaria de temperatura (bulbo seco y bulbo húmedo) para un dìa de cálculo de carga de enfriamiento o para estimaciones de consumo de energía. Con los resultados obtenidos conviene efectuar este proceso mediante factores horarios de corrección basados en la temperatura para un porcentaje de diseño y ámbito diario y, en el caso de bulbo húmedo, suponiendo que el punto de rocío se mantiene constante; ésto puede hacerse para cada estación y para cada mes del año.

e- Entre los aspectos que pueden mejorar la información descrita, o su utlización, están:

1- Aumentar los tamaños de las muestras de datos de algunas de las estaciones estudiadas empleando información recopilada recientemente.

2- Obtener curvas horarias de temperaturas para análisis de consumo de energía en las estaciones en donde la información lo permita.

3- Emplear "software" salido recientemente, para mejorar los métodos de procesamiento de los datos.

\section{BIBLIOGRAFIA}

1- Arias A. Jorge, Alvarez C. Juan. Obtención de condiciones externas de diseño para aire acondicionado. Trabajo Final de Graduación, Escuela de Ingeniería Mecánica, Universidad de Costa Rica, 1982. 
2- ASHRAE Handbook, 1985 Fundamentals, cap 24. American Society of Heating, Refrigerating and Air conditioning Engineers, Inc. Atlanta, Georgia, EE.UU.

3- Bolaños, Ronald. Análisis de información meteorológica para diseño de sistemas térmicos. Trabajo Final de Graduación, Escuela de Ingeniería Mecánica, Universidad de Costa Rica, 1986.

4- Bonilla M., Javier. Procedimiento para determinar el comportamiento de la temperatura y la humedad relativa en una localidad utilizando un microcomputador personal. Trabajo Final de Graduación, Escuela de Ingeniería Mecánica, Universidad de Costa Rica, 1989.

5- Boyd, Donald. Chairman TC-4-2, Weather Data and Design Conditions. American Society of Heating, Refrigerating and Air conditioning Engineers. Comunicación personal, julio 1974.

6- Carrier Air Conditionig Company, System Design Manual. Syracuse, New York, EE.UU. Eigth Printing, 1968.

7- Carrier Air Conditioning Company, E-20.

8- Garro R. Olman. Determinación de los parámetros ambientales de diseño para sistemas térmicos en Pavas. Trabajo Final de Graduación, Escuela de Ingeniería Mecánica, Universidad de Costa Rica, 1991
9- Gonzalez M. Javier, Alpizar S. Martín. Obtención de condiciones externas de diseño para aire acondicionado II parte. Trabajo Final de Graduación, Escuela de Ingeniería Mecánica, Universidad de Costa Rica, 1982.

10- Holladay William, editor. Recommended Outdoor Design Temperatures Southern California, Arizona, Nevada. American Society of Heating, Refrigerating and Air conditioning Engineers, Inc., Southern California and San Diego Chapters, 1972.

11- Holladay, William 1. Suggested Revision of Weather -Data Design Temperatures. ASHRAE Journal, September 1973.

12- Marley Company. Cooling Tower Fundamentals. Fecha aproximada 1973. Kansas City, Missouri EE.UU.

13- Peralta G. Teófilo. Obtención de condiciones externas de diseño para aire acondicionado, IV parte. Trabajo Final de Graduación, Escuela de Ingeniería Mecánica, Universidad de Costa Rica, 1983.

14- Ramirez C. Juan Carlos. Determinación de los parámetros ambientales de diseño para sistemas de aire acondicionado en Liberia. Trabajo Final de Graduación, Escuela de Ingeniería Mecánica, Universidad de Costa Rica, 1990. 